\title{
Zusätzliche Arztleistungen zulasten der Zusatzversicherungen bei Spitalaufenthalt
}

\author{
B. Burri *
}

Unter dem System des alten KUVG, also vor der Einführung des KVG 1996, kannten die Grundversicherungen und die Zusatzversicherungen weitgehend analoge Strukturen und Rechtsformen, was freilich auch zu etlichen Schwierigkeiten führte, die die Juristen beschäftigten. Mit dem neuen Gesetz, insbesondere Artikel 12, Absatz 3 KVG, hat der Gesetzgeber gewollt oder ungewollt eine klare Trennung für die zwei Bereiche der Krankenversicherung, wie wir sie hierzulande kennen, herbeigeführt: Der eine, Sozialoder Grundversicherung genannt und obligatorisch, wird vom KVG bestimmt und ist dem öffentlichen Recht zugeordnet. Der andere, Zusatzversicherung genannt und freiwillig, soll definitionsgemäss die soziale Grundversicherung ergänzen. Dieser zweite Bereich bestimmt sich nach dem Versicherungsvertragsgesetz (VVG) und damit nach Privatrecht.

Die normative Separierung hat zahlreiche Konsequenzen, deren Auswirkungen im einzelnen bislang nicht überall erkannt sind. Die juristische Zweiteilung betrifft nicht nur alle Akteure im Gesundheitswesen, vom Patienten über die Versicherer bis zu den Leistungserbringern, sondern berührt ebenso die Pflegeleistungen und namentlich deren Finanzierung, was bekanntlich eines der zentralen Anliegen der 2. KVGRevision ist.

Diese Zweiteilung betrifft hauptsächlich die Leistungen bei Spitalaufenthalt, und zwar unabhängig davon, ob dieser in einer nicht subventionierten Privatklinik oder in der Privatabteilung eines öffentlichen Spitals erfolgt. Die Spitalleistungen unterteilen sich zum einen in solche, die von der sozialen Grundversicherung übernommen werden. Sie sind im Gesetz (Artikel 25-31) aufgezählt und ihre Kostenübernahme ist in Artikel 32 KVG geregelt. Zum andern geht es um Leistungen, die eben diesen Kriterien nicht entsprechen und «Nicht-KVG-Leistungen» genannt werden. Letztere können von der privaten Spitalzusatzversicherung übernommen werden. Allerdings sind die Grenzen zwischen diesen beiden Kategorien von Spitalleistungen in der Praxis in verschiedener Hinsicht noch wenig klar und bedürfen - mangels Entscheidungen auf politischer Ebene - zur Verdeutlichung vermutlich noch etlicher Gerichtsentscheide.
Unsere ärztliche Tätigkeit setzt sich naturgemäss aus verschiedenen Pflegekomponenten zusammen. Sie wird damit auch von dieser Separierung zwischen sozialer Grundversicherung und Spitalzusatzversicherung erfasst, ob die Behandlung nun in einem nicht subventionierten Privatspital oder der Privat-/Halbprivatabteilung eines öffentlichen oder öffentlich-subventionierten Krankenhauses erfolgt. Ein Teil unserer ärztlichen Tätigkeit im Spital geht zu Lasten der sozialen Grundversicherung, während ergänzende Leistungen zu Lasten des Patienten oder seiner Zusatzversicherung gehen.

Für unsere Vereinigung erschien es von besonderer Bedeutung, für die Belegärzte den zusätzlichen ärztlichen Leistungsteil - eben ausserhalb des KVG-Bereiches - in einem nichtsubventionierten Privatspital zu umschreiben. Unsere entsprechenden Gedanken und Überlegungen wurden durch ein von Professor Dr. Robert Leu im Jahr 2000 erstelltes Gutachten angeregt. Er hatte darin die zunehmenden Schwierigkeiten der Versicherten aufgezeigt, zwischen diesen zwei Versicherungsgebieten unterscheiden zu können. Auch die Schweizerische Patientenorganisation brachte dieses Thema in den letzten Monaten zur Sprache. Zu Recht wollte man erfahren, was eigentlich die Zusatzleistungen sind und speziell, welche zusätzlichen Elemente der Patient vom Belegarzt erwarten darf, wenn er eine Zusatzversicherung mit der Überlegung abgeschlossen hat, im Falle einer Spitalbehandlung auch die freie Arztwahl zu haben.

Die Schweizerische Vereinigung der Belegärzte an Privatkliniken hat sich nach eingehenden Beratungen an ihrer Generalversammlung sowie in entsprechenden Diskussionen mit der Schweizerischen Patientenorganisation auf einige Komponenten medizinischer Zusatzleistungen festgelegt, die der Patient mit einer Privat-/Halbprivatversicherung bei Spitalbehandlungen geltend machen kann. Die Belegärzte an Privatkliniken sind der Auffassung, dass der Bereich der ärztlichen Versorgung in der Zusatzversicherung im Falle einer Spitalbehandlung auf der Privat- oder Halbprivatabteilung auf den folgenden acht Punkten beruht:

- Der vom Patienten frei gewählte Arzt erbringt die ärztlichen Leistungen persönlich. 
- Die Ausbildung des Spezialarztes ist abgeschlossen.

- Der Arzt ist permanent verfügbar und der erleichterte Zugang zu ihm ermöglicht eine persönliche therapeutische Begleitung.

- Der Arzt misst den Wünschen des Patienten hinsichtlich des Ablaufs der Behandlung grosse Bedeutung bei.

- Der Arzt achtet speziell auf den Schutz der Privatsphäre des Patienten.

- Der Arzt erstellt eine transparente Honorarrechnung.

- Der Arzt anerkennt die Paritätische Kommission der Zusatzversicherungen und deren Spielregeln.

- Ein eventueller ärztlicher Stellvertreter oder beauftragter Arzt erfüllt die gleichen Bedingungen.
Dieses 8-Punkte-Programm erscheint unseres Erachtens klar, mit Ausnahme vielleicht vom siebten Punkt, der wohl erst in einigen Kantonen der Westschweiz realisiert wird.

Allgemein ist erwiesen, dass die 1996 eingeführte neue Regelung viele Probleme im schweizerischen Gesundheitswesen geschaffen hat. Die vom KVG nun bestimmte klare Trennung zwischen den seit langen Jahren eingeführten zwei Versicherungskomponenten greift jetzt Platz. Die Schweizerische Vereinigung der Belegärzte an Privatkliniken hofft, mit dem 8-Punkte-Programm zur Klärung der Situation beizutragen, was unsere ärztliche Tätigkeit im Spital betrifft. Gleichzeitig soll damit auch das Verständnis für das Funktionieren und die Vorteile der Zusatzversicherungen bei Versicherten und Patienten gefördert werden.

\title{
Complément médical en cas d'hospitalisation à charge de l'assurance complémentaire
}

\author{
B. Burri *
}

\footnotetext{
* Président de l'Association suisse des médecins indépendants travaillant en cliniques privées ASMI/SVBP
}

\section{Correspondance:}

Dr Bernard Burri

Moosstrasse 2

Case postale 29

CH-3073 Gümligen-Berne

Tél. 0319527905

Fax 0319527683

e-mail: uwanner@swissonline.ch
Sous le régime de la LAMA, donc avant l'introduction de la LAMal en 1996, l'assurance de base et les assurances complémentaires avaient un fonctionnement et un statut juridique proches l'un de l'autre, ce qui n'était pas sans créer quelques difficultés que les juristes avaient stigmatisées. Avec la nouvelle loi - il s'agit de l'article 12 alinéa 3 de la LAMal - le législateur a, à tort ou à raison, clairement séparé les deux domaines de l'assurance maladie que nous connaissons dans notre pays: l'un, obligatoire, appelé assurance sociale ou de base, est régi par la LAMal et relève par conséquent du droit public; l'autre, facultatif, dit assurance complémentaire - devant par définition compléter l'assurance sociale - est versé dans le droit privé et soumis ainsi à la loi fédérale sur le contrat d'assurance (LCA). Cette dissociation juridique a des conséquences multiples dont l'ampleur de certaines n'est probablement pas encore perçue à sa juste valeur. Elle concerne non seulement tous les acteurs de la santé que sont les patients, les assureurs et les fournisseurs de soins mais également les soins dans leur étendue et surtout dans leur financement, ce dernier point étant, comme cela est connu de tous, l'un des enjeux de la $2^{\mathrm{e}}$ révision de la LAMal.

Cette séparation concerne les soins prodigués en cas d'hospitalisation principalement, que cela soit dans un établissement privé non subventionné ou dans une division privée d'un établissement subventionné. Ces soins se clivent d'une part en prestations prises en charge par l'assurance sociale - elles sont énumérées dans les articles 25 à 31 et les conditions de prise en charge sont définies dans l'art. 32 de la LAMaL d'autre part en celles ne répondant pas à ces critères - appelées alors prestations hors LAMal pouvant alors être prises en compte par l'assurance complémentaire d'hospitalisation. Dans la pratique, la frontière entre les deux types de prestations est encore incertaine à plus d'un égard et nécessitera encore plusieurs décisions de tribunal à défaut de décision politique.

Notre activité médicale est naturellement une composante des soins et est, par conséquent, éga- 
lement soumise à la séparation entre l'assurance sociale et l'assurance complémentaire en cas d'hospitalisation que cela soit dans un établissement privé non subventionné ou dans une division privée d'un établissement subventionné. Une part de notre activité médicale hospitalière est à charge de l'assurance sociale alors que son complément est de la responsabilité du patient ou de son assurance complémentaire. Il a paru primordial à notre Association de définir la part médicale complémentaire - donc hors LAMal - de notre activité en tant que médecin agréé en cas d'hospitalisation dans un établissement privé non subventionné. Notre réflexion à ce sujet a été stimulée par l'expertise publiée en 2000 du Prof. R. Leu, qui avait signalé la difficulté croissante des assurés à distinguer les deux domaines d'assurance maladie et, surtout ces deniers mois, par l'Organisation suisse des patients qui, à juste raison, a souhaité connaître le complément des soins et, plus précisément en ce qui nous concerne, le complément médical que peut obtenir du médecin agréé un assuré lorsqu'il a contracté une assurance complémentaire pour motif d'avoir le libre choix du médecin en cas d'hospitalisation.

L'Association suisse des médecins indépendants travaillant en cliniques privées, après en avoir débattu lors de sa dernière assemblée générale et longuement discuté avec l'Organisation suisse des patients, a retenu un certain nombre d'éléments qui définissent la part médicale complémentaire à laquelle le patient a droit lorsqu'il a conclu une assurance complémentaire en cas d'hospitalisation. Les médecins agréés en établissements privés non subventionnés sont d'avis que le contenu médical de l'assurance complémentaire en cas d'hospitalisation (qu'elle soit privée ou semi-privée) repose sur les huit points suivants:
- le médecin librement choisi par le patient réalise personnellement les prestations médicales;

- la formation de médecin spécialiste est achevée;

- la disponibilité du médecin est permanente et son accessibilité aisée assure un accompagnement thérapeutique personnel;

- le médecin attache une grande importance au souhait du patient dans l'organisation de la prestation;

- le médecin veille particulièrement à la protection de la sphère privée du patient;

- le médecin établit une note d'honoraires transparente et compréhensible;

- le médecin accepte les règles de fonctionnement et la commission paritaire, lorsqu'elles existent, des assurances complémentaires;

- l'éventuel médecin remplaçant ou mandaté répond à ces mêmes critères.

Les huit points du complément médical énumérés ci-dessus sont à notre avis explicites, sauf le septième qui est connu pour le moment uniquement dans certains cantons de Suisse romande.

C'est une évidence pour tous que l'introduction de la nouvelle réglementation en 1996 a créé beaucoup de difficultés dans le paysage de la santé en Suisse. La séparation nette exigée par la LAMal des deux domaines d'assurances maladies que nous connaissons de longue date est en passe de se mettre en place. L'association suisse des médecins travaillant en cliniques privées espère ainsi avoir participé à la clarification de la situation en ce qui concerne notre activité médicale hospitalière et en cela faciliter la compréhension du fonctionnement des assurances complémentaires pour les assurés et les patients. 